\title{
Swarm Intelligence-Based Smart Energy Allocation Strategy for Charging Stations of Plug-In Hybrid Electric Vehicles
}

\author{
Imran Rahman, ${ }^{1}$ Pandian M. Vasant, ${ }^{1}$ \\ Balbir Singh Mahinder Singh, ${ }^{1}$ and M. Abdullah-Al-Wadud ${ }^{2}$ \\ ${ }^{1}$ Department of Fundamental and Applied Sciences, Universiti Teknologi PETRONAS, Bandar Seri Iskandar, \\ 31750 Tronoh, Perak, Malaysia \\ ${ }^{2}$ Department of Software Engineering, College of Computer and Information Sciences, King Saud University, \\ P.O. BOX 2454, Riyadh 11451, Saudi Arabia
}

Correspondence should be addressed to Pandian M. Vasant; pvasant@gmail.com

Received 15 May 2014; Accepted 18 August 2014

Academic Editor: Baozhen Yao

Copyright (C) 2015 Imran Rahman et al. This is an open access article distributed under the Creative Commons Attribution License, which permits unrestricted use, distribution, and reproduction in any medium, provided the original work is properly cited.

\begin{abstract}
Recent researches towards the use of green technologies to reduce pollution and higher penetration of renewable energy sources in the transportation sector have been gaining popularity. In this wake, extensive participation of plug-in hybrid electric vehicles (PHEVs) requires adequate charging allocation strategy using a combination of smart grid systems and smart charging infrastructures. Daytime charging stations will be needed for daily usage of PHEVs due to the limited all-electric range. Intelligent energy management is an important issue which has already drawn much attention of researchers. Most of these works require formulation of mathematical models with extensive use of computational intelligence-based optimization techniques to solve many technical problems. In this paper, gravitational search algorithm (GSA) has been applied and compared with another member of swarm family, particle swarm optimization (PSO), considering constraints such as energy price, remaining battery capacity, and remaining charging time. Simulation results obtained for maximizing the highly nonlinear objective function evaluate the performance of both techniques in terms of best fitness.
\end{abstract}

\section{Introduction}

The vehicular network recently accounts for around $25 \%$ of $\mathrm{CO}_{2}$ emissions and over $55 \%$ of oil consumption around the world [1]. Carbon dioxide $\left(\mathrm{CO}_{2}\right)$ is the primary greenhouse gas emitted through human activities like combustion of fossil fuels (coal, natural gas, and oil) for energy and transportation. Several researchers have proved that a great amount of reductions in greenhouse gas emissions and the increasing dependence on oil could be accomplished by electrification of transport sector [2]. Indeed, the adoption of hybrid electric vehicles (HEVs) has brought significant market success over the past decade. Vehicles can be classified into three groups: internal combustion engine vehicles (ICEVs), hybrid electric vehicles (HEVs), and all-electric vehicles (AEVs) [3]. Plugin hybrid electric vehicles (PHEVs) which are very recently introduced promise to boost up the overall fuel efficiency by holding a higher capacity battery system, which can be directly charged from traditional power grid system that helps the vehicles to operate continuously in "all-electric range" (AER). All-electric vehicle or AEV is a vehicle using electric power as the only source to move the vehicle [4]. Plug-in hybrid electric vehicles with a connection to the smart grid can possess all of these strategies. Hence, the widely extended adoption of PHEVs might play a significant role in the alternative energy integration into traditional grid systems [5]. There is a need of efficient mechanisms and algorithms for smart grid technologies in order to solve highly heterogeneous problems like energy management, cost reduction, efficient charging infrastructure, and so forth with different objectives and system constraints [6].

According to a statistics of Electric Power Research Institute (EPRI), about $62 \%$ of the entire United States (US) vehicle will comprise PHEVs within the year 2050 [7]. 
Moreover, there is an increasing demand to implement this technology on the electric grid system. Large numbers of PHEV s have the capability to threaten the stability of the power system. For example, in order to avoid interruption when several thousand PHEVs are introduced into the system over a short period of time, the load on the power grid will need to be managed very carefully. One of the main targets is to facilitate the proper interaction between the power grid and the PHEV. For the maximization of customer satisfaction and minimization of burdens on the grid, a complicated control mechanism will need to be addressed in order to govern multiple battery loads from a number of PHEVs appropriately [8]. The total demand pattern will also have an important impact on the electricity industry due to differences in the needs of the PHEV s parked in the deck at certain time [9]. Proper management can ensure strain minimization of the grid and enhance the transmission and generation of electric power supply. The control of PHEV charging depending on the locations can be classified into two groups: household charging and public charging. The proposed optimization focuses on the public charging station for plug-in vehicles because most of PHEV charging is expected to take place in public charging locations [10].

Wide penetration of PHEVs in the market depends on a well efficient charging infrastructure. The power demand from this new load will put extra stress on the traditional power grid [11]. As a result, a good number of PHEV charging infrastructures with appropriate facilities are essential to be built for recharging electric vehicles; for this some strategies have been proposed by the researchers $[12,13]$. Charging stations are needed to be built at workplaces, markets/shopping malls, and home. In [14], authors proposed the necessity of building new smart charging station with effective communication among utilities along with substation control infrastructure in view of grid stability and proper energy utilization. Furthermore, assortment of charging stations with respect to charging characteristics of different PHEVs traffic mobility characteristics, sizeable energy storage, cost minimization, quality of services (QoS), and optimal power of intelligent charging station are underway [15]. Thus, evolution of reliable, efficient, robust, and economical charging infrastructure is underway. In this wake, numerous techniques and methods have been proposed for deployment of charging station for PHEVs $[16,17]$.

One of the important constraints for accurate charging is state of charge (SoC). Charging algorithm can accurately be managed by the precise state of charge estimation [18]. An approximate graph of a typical lithium-ion cell voltage versus SoC is shown in Figure 1. The figure indicates that the slope of the curve below $20 \%$ and above $90 \%$ is high enough to result in a detectable voltage difference to be relied on by charge balancing control and measurement circuits [19]. There is a need of in-depth study on maximization of average SoC in order to facilitate intelligent energy allocation for PHEVs in a charging station. Gravitational search algorithm (GSA) is one of the newest heuristic algorithms introduced by Rashedi et al. [20]. GSA algorithm is also a member of swarm intelligence family which is inspired by the well-known law of

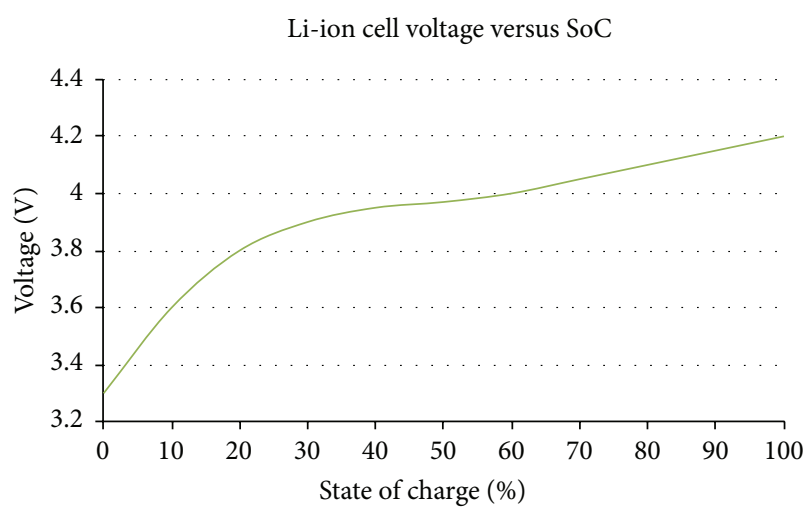

Figure 1: Li-ion cell voltage versus state of charge [24].

gravity and interactions between the masses and implements Newtonian gravity and the laws of motion [21-23].

GSA-based optimization has already been used by the researchers for postoutage bus voltage magnitude calculations, economic dispatch with valve-point effects, optimal sizing and suitable placement for distributed generation (DG) in distribution system, optimization of synthesis gas production [25], rectangular patch antenna [26], orthogonal array based performance improvement [27], solving thermal unit commitment (UC) problem, and finding out optimal solution for optimal power flow (OPF) problem in a power system [28]. Specifically, we are investigating the use of the gravitational search algorithm (GSA) method for developing real-time and large-scale optimizations for allocating power.

The remainder of this paper is organized as follows: next section will describe the specific problem that we are trying to solve. We will provide the optimization objective and constraints and mathematical formulation of our algorithm, review the GSA method, and describe how the algorithm works for our optimization problems. The simulation results and analysis are then presented. Finally, conclusions and future directions are drawn.

\section{Problem Formulation}

The idea behind smart charging is to charge the vehicle when it is most beneficial such as when the electricity price and total power demand remain lowest or there is excess capacity of generated power [24].

Suppose there is a charging station with the capacity of total power $P$. Total $N$ numbers of PHEVs need to be charged within 24 hours of time interval. The proposed system should allow PHEVs to leave the charging station before their expected leaving time for making the system more effective. It is worth to mention that each PHEV is regarded to be plugged in to the charging station once. The main aim is to allocate power intelligently for each PHEV coming to the charging station. The state of charge is the main parameter which needs to be maximized in order to allocate power effectively. For this, the objective function considered in this paper is the maximization of average SoC and thus allocates energy for PHEVs at the next time step. 
The constraints considered are charging time, present SoC, and price of the energy.

The objective function is defined as

$$
\begin{aligned}
\max J(k) & =\sum_{i} w_{i}(k) \operatorname{SoC}_{i}(k+1), \\
w_{i}(k) & =f\left(C_{r, i}(k), T_{r, i}(k), D_{i}(k)\right), \\
C_{r, i}(k) & =\left(1-\operatorname{SoC}_{i}(k)\right) * C_{i},
\end{aligned}
$$

where $C_{r, i}(k)$ is the battery capacity (remaining) needed to be filled for $i$ number of PHEV at time step $k$; $C_{i}$ is the battery capacity (rated) of the $i$ number of PHEV; remaining time for charging a particular PHEV at time step $k$ is expressed as $T_{r, i}(k)$; the price difference between the real-time energy price and the price that a specific customer at the $i$ number of PHEV charger is willing to pay at time step $k$ is presented by $D_{i}(k) ; w_{i}(k)$ is the charging weighting term of the $i$ number of PHEV at time step $k$ (a function of charging time, present SoC, and price of the energy); $\mathrm{SoC}_{i}(k+1)$ is the state of charge of the $i$ number of PHEV at time step $k+1$.

Here, the weighting term indicates a bonus proportional to the attributes of a specific PHEV. For example, if a PHEV has a lower initial SoC and less charging time (remaining), but the driver is eager to pay a higher price, the system will provide more power to this particular PHEV battery charger:

$$
w_{i}(k) \alpha\left[\operatorname{Cap}_{r, i}(k)+D_{i}(k)+\frac{1}{T_{r, i}}(k)\right] .
$$

The charging current is also assumed to be constant over $\Delta t$ :

$$
\begin{gathered}
{\left[\operatorname{SoC}_{i}(k+1)-\operatorname{SoC}_{i}(k)\right] \cdot \operatorname{Cap}_{i}=Q_{i}=I_{i}(k) \Delta t} \\
\operatorname{SoC}_{i}(k+1)=\operatorname{SoC}_{i}(k)+\frac{I_{i}(k) \Delta t}{\operatorname{Cap}_{i}}
\end{gathered}
$$

where the sample time $\Delta t$ is defined by the charging station operators and $I_{i}(k)$ is the charging current over $\Delta t$.

The battery model is regarded as a capacitor circuit, where $C_{i}$ is the capacitance of battery (Farad). The model is defined as

$$
C_{i} \cdot \frac{d V_{i}}{d t}=I_{i}
$$

Therefore, over a small time interval, one can assume the change of voltage to be linear:

$$
\begin{gathered}
C_{i} \cdot \frac{\left[V_{i}(k+1)-V_{i}(k)\right]}{\Delta t}=I_{i}, \\
V_{i}(k+1)-V_{i}(k)=\frac{I_{i} \Delta t}{C_{i}} .
\end{gathered}
$$

As the decision variable used here is the allocated power to the PHEVs, by replacing $I_{i}(k)$ with $P_{i}(k)$ the objective function finally becomes

$$
\begin{array}{r}
J(k)=\sum w_{i} \cdot\left[\operatorname{SoC}_{i}(k)+\left(2 P_{i}(k) \Delta t\right)\right. \\
\times\left(0 . 5 \cdot C _ { i } \cdot \left[\sqrt{\frac{2 P_{i}(k) \Delta t}{C_{i}}+V_{i}^{2}(k)}\right.\right. \\
\left.\left.\left.+V_{i}(k)\right]\right)^{-1}\right] .
\end{array}
$$

2.1. System Constraints. Possible real-world constraints could include the charging rate (i.e., slow, medium, and fast), the time that the PHEV is connected to the grid, the desired departure SOC, the maximum electricity price that a user is willing to pay, and certain battery requirements. Furthermore, the available communication bandwidth could limit sampling time, which would have effects on the processing ability of the vehicle.

Power obtained from the utility $\left(P_{\text {utility }}\right)$ and the maximum power $\left(P_{i, \max }\right)$ absorbed by a specific PHEV are the primary energy constraints being considered in this paper. The power demand of a PHEV/PEV cannot exceed the rated power output of the battery charger:

$$
\begin{gathered}
\sum_{i} P_{i}(k) \leq P_{\text {utility }}(k) \times \eta, \\
0 \leq P_{i}(k) \leq P_{i, \text { max }}(k) .
\end{gathered}
$$

The overall charging efficiency of a particular charging infrastructure is described by $\eta$. From the system point of view, charging efficiency is supposed to be constant at any given time step. Maximum battery SoC limit for the $i$ number of PHEV is $\mathrm{SoC}_{i, \max }$. When $\mathrm{SoC}_{i}$ reaches the values close to SoC $_{i, \max }$, the $i$ number of battery charger shifts to a standby mode. The state of charge ramp rate is confined within limits by the constraint $\Delta \mathrm{SoC}_{\max }$. To accommodate the system dynamics, the energy scheduling is updated when when (i) system utility data is updated; (ii) a new PHEV is plugged in, and (iii) time period $\Delta t$ has periodically passed. Table 1 shows all the objective function parameters that were tuned for performing the optimization.

Energy allocation to PHEV charging station is subjected to various constraints as mentioned in the problem formulation section. Different constraints make the entire search space limited to a particular suitable region. So, a powerful optimization algorithm should be implemented in order to achieve high quality solutions with a stable convergence rate.

\section{Gravitational Search Algorithm}

GSA is an optimization method which has been introduced by Rashedi et al. in the year of 2009 [20]. In GSA, the 
TABLE 1: Parameter settings of the objective function.

\begin{tabular}{ll}
\hline Parameter & Values \\
\hline \multirow{2}{*}{$\begin{array}{l}\text { Fixed } \\
\text { parameters }\end{array}$} & Charimum power, $P_{i, \max }=6.7 \mathrm{kWh}$ \\
& Total charging time, $\Delta t=20$ minutes \\
& (1200 seconds) \\
& Power allocation to each PHEV: $30 \mathrm{~W}$ \\
\hline & $0.2 \leq$ state of charge $(\mathrm{SoC}) \leq 0.8$ \\
& Waiting time $\leq 30$ minutes $(1800$ \\
& seconds $)$ \\
Variables & $16 \mathrm{kWh} \leq$ battery capacity $\left(C_{i}\right) \leq$ \\
& $40 \mathrm{kWh}$ \\
\hline & $\sum P_{i}(k) \leq P_{\text {utility }}(k) \times \eta$ \\
& $0 \leq P_{i}(k) \leq P_{i, \max }(k)$ \\
& $0 \leq \operatorname{SoC}_{i}(k) \leq \operatorname{SoC}_{i, \max }$ \\
& $0 \leq \operatorname{SoC}_{i}(k+1)-\operatorname{SoC}_{i}(k) \leq \Delta \operatorname{SoC}_{\max }$ \\
\hline
\end{tabular}

specifications of each mass (or agent) are four in total, which are inertial mass, position, active gravitational mass and passive gravitational mass. The position of the mass presents a solution of a particular problem and masses (gravitational and inertial) are obtained by using a fitness function. GSA can be considered as a collection of agents (candidate solutions), whose masses are proportional to their value of fitness function. During generations, all masses attract each other by the gravity forces between them. A heavier mass has the bigger attraction force. Therefore the heavier masses which are probably close to the global optimum attract the other masses proportional to their distances.

3.1. Law of Gravity. The law states that particles attract each other and the force of gravitation between two particles is directly proportional to the product of their masses and inversely proportional to the distance between them.

3.2. Law of Motion. The law states that the present velocity of any mass is the summation of the fraction of its previous velocity and the velocity variance. Variation in the velocity or acceleration of any mass is equal to the force divided by inertia mass.

The gravitational force is expressed as follows:

$$
F_{i j}^{d}(t)=G(t) \frac{M_{p i}(t) \times M_{a j}(t)}{R_{i j}(t)+\varepsilon}\left(x_{j}^{d}(t)-x_{i}^{d}(t)\right),
$$

where $M_{a j}$ is the active gravitational mass related to agent $j$, $M_{p i}$ is the passive gravitational mass related to agent $i, G(t)$ is gravitational constant at time $t, \varepsilon$ is a small constant, and $R_{i j}(t)$ is the Euclidian distance between two agents $i$ and $j$. The $G(t)$ is calculated as follows:

$$
G(t)=G_{0} \times \exp \left(\frac{-\alpha \times \text { iter }}{\text { max iter }}\right),
$$

where $\alpha$ and $G_{0}$ are descending coefficient and primary value, respectively, and current iteration and maximum number of iterations are expressed as iter and max iter. In a problem space with the dimension $d$, the overall force acting on agent $i$ is estimated as the following equation:

$$
F_{i}^{d}(t)=\sum_{j=1, j \neq i}^{N} \operatorname{rand}_{j} F_{i j}^{d}(t),
$$

where $\operatorname{rand}_{j}$ is a random number with interval $[0,1]$. From law of motion we know that an agent's acceleration is directly proportional to the resultant force and inverse of its mass, so the acceleration of all agents should be calculated as follows:

$$
\operatorname{ac}_{i}^{d}(t)=\frac{F_{i}^{d}(t)}{M_{i i}(t)}
$$

where $t$ is a specific time and $M_{i i}$ is the mass of the object $i$. The velocity and position of agents are calculated as follows:

$$
\begin{gathered}
\operatorname{vel}_{i}^{d}(t+1)=\operatorname{rand}_{i} \times \operatorname{vel}_{i}^{d}(t)+\operatorname{ac}_{i}^{d}(t), \\
x_{i}^{d}(t+1)=x_{i}^{d}(t)+\operatorname{vel}_{i}^{d}(t+1),
\end{gathered}
$$

where $\operatorname{rand}_{i}$ is a random number with interval $[0,1]$.

Gravitational and inertia masses are simply calculated by the fitness evaluation. A heavier mass means a more efficient agent. This means that better agents have higher attractions and walk more slowly. Assuming the equality of the gravitational and inertia mass, the values of masses are calculated using the map of fitness. We update the gravitational and inertial masses by the following equations:

$$
M_{a i}=M_{p i}=M_{i i}=M_{i}, \quad i=1,2, \ldots, N .
$$

In gravitational search algorithm, all agents are initialized first with random values. Each of the agents is a candidate solution. After initialization, velocities for all agents are defined using (12). Moreover, the gravitational constant, overall forces, and accelerations are determined by (9), (10), and (11), respectively. The positions of agents are calculated using (13). At the end, GSA will be terminated by meeting the stopping criterion of maximum 100 iterations. The parameter settings for GSA are demonstrated in Table 2. Moreover, GSA flowchart is shown in Figure 2.

\section{Simulation Results and Analysis}

The GSA algorithm was applied to find out global best fitness of the objective function (Algorithm 1). All the calculations were run on an Intel (R) Core i5-3470 M CPU@ 3.20 GHz, $4.00 \mathrm{~GB}$ RAM, Microsoft 32 bit Windows 7 OS, and MATLAB $^{\odot}$ R2013a.

Many optimization algorithms involve local search techniques which can get stuck on local maxima. Most search techniques strive to find a global maximum in the presence of local maxima [29]. One of the most important characteristics of GSA is its significant performance during exploration process. The capability of an algorithm to extend the problem in search gap is known as exploration while the ability of an algorithm to recognize optimal solution near a favorable one is exploitation [30,31]. 


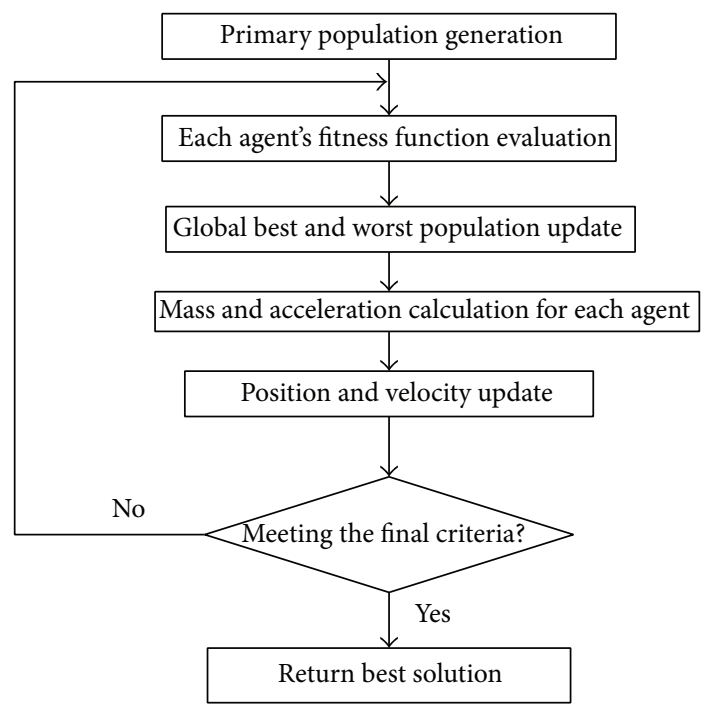

FIGURE 2: The GSA flowchart.

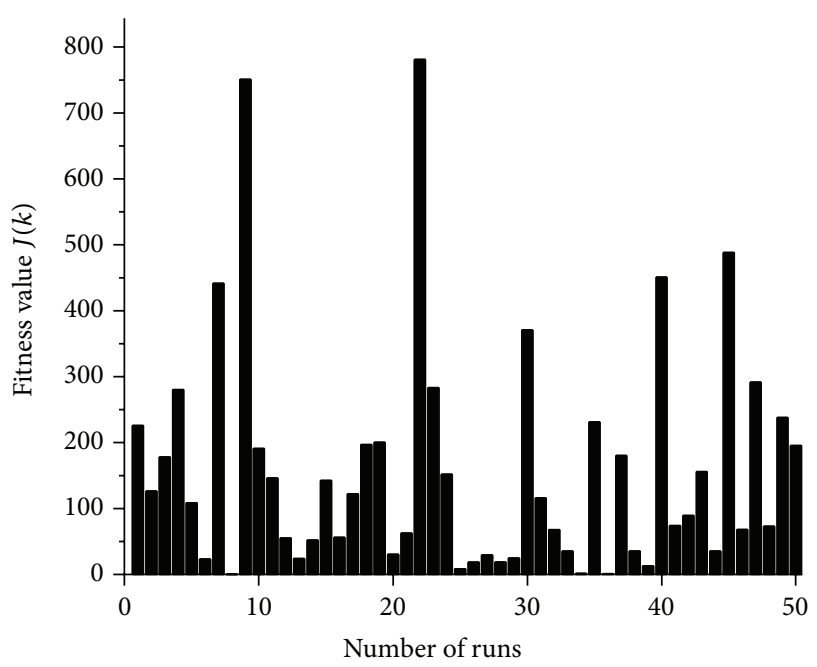

Figure 3: Fitness value versus number of runs (50 PHEVs).

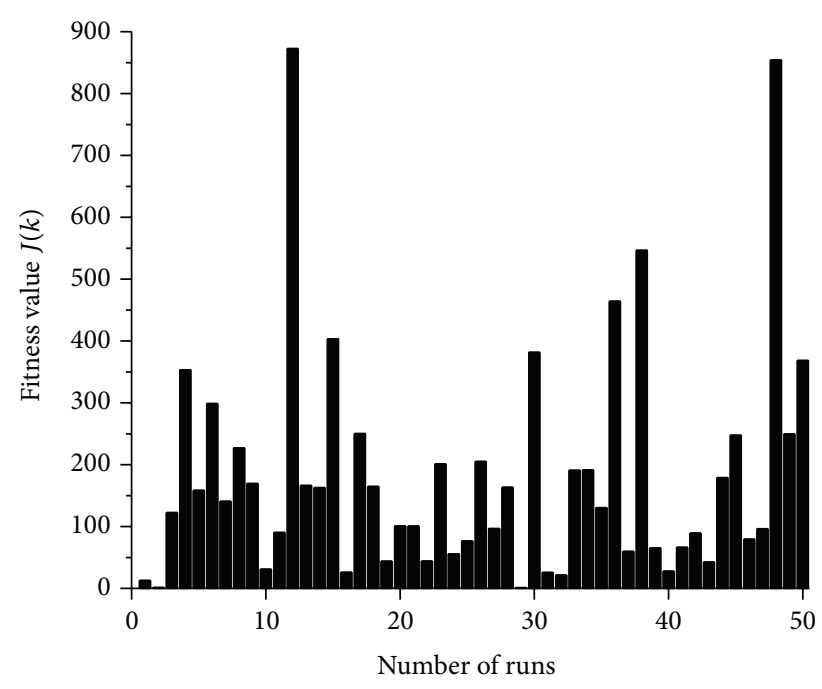

FIGURE 4: Fitness value versus number of runs (100 PHEVs).

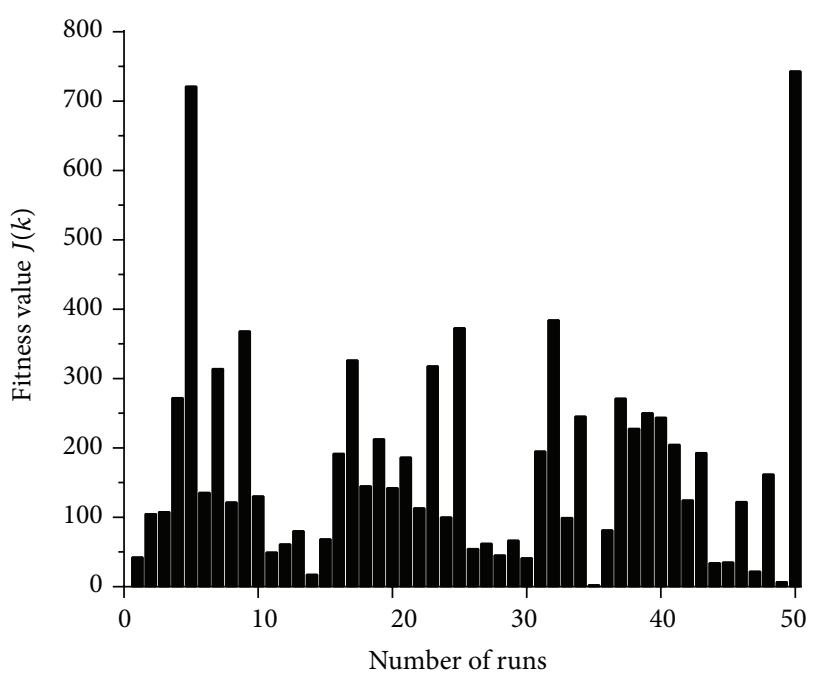

FIGURE 5: Fitness value versus number of runs (300 PHEVs).

TABLE 2: GSA parameter settings.

\begin{tabular}{lc}
\hline Parameters & Values \\
\hline Primary parameter, $G_{0}$ & 100 \\
Number of mass agents, $n$ & 100 \\
Constant parameter, $\alpha$ & 20 \\
Constant parameter, $\varepsilon$ & .01 \\
Power of " $R$ " & 1 \\
Maximum iteration & 100 \\
Number of runs & 50 \\
\hline
\end{tabular}

Figures 3, 4, 5, 6, and 7 show the simulation results for $50,100,300,500$, and 1000 plug-in hybrid electric vehicles (PHEVs), respectively, for finding the maximum fitness value of objective function J. In order to evaluate the performance and show the efficiency and superiority of the proposed algorithm, we ran each scenario 50 times in total.

For Figure 3 (50 PHEVs), the maximum best fitness and minimum best fitness were 781.1267 and 0.2191 , respectively.

The average best fitness is 158.8289. Figure 4 depicts the maximum fitness value for 100 PHEVs. In this case, the maximum best fitness and minimum best fitness were 579.3955 and 3.2523. The average best fitness is decreased into 139.7536.

For Figure 5 (300 PHEVs), the maximum best fitness and minimum best fitness were 743.1251 and 2.3279 , respectively. The average best fitness is 172.4296 .

Figure 6 depicts the maximum fitness value for 500 PHEVs. In this case, the maximum best fitness and minimum best fitness were 836.2707 and 0.9818 . The average best fitness is decreased into 152.36437.

Figure 7 shows the maximum fitness value for 1000 PHEVs. In this case, the maximum best fitness and minimum best fitness were 968.7652 and 7.2747. The average best fitness is decreased into 161.52349 . 


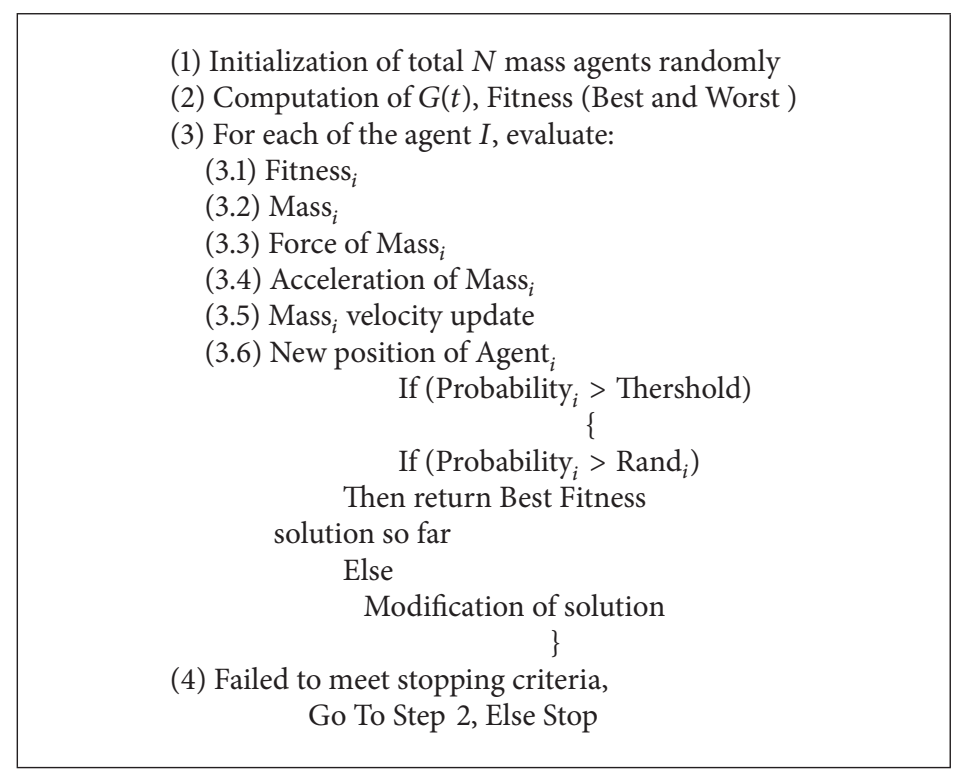

Algorithm 1

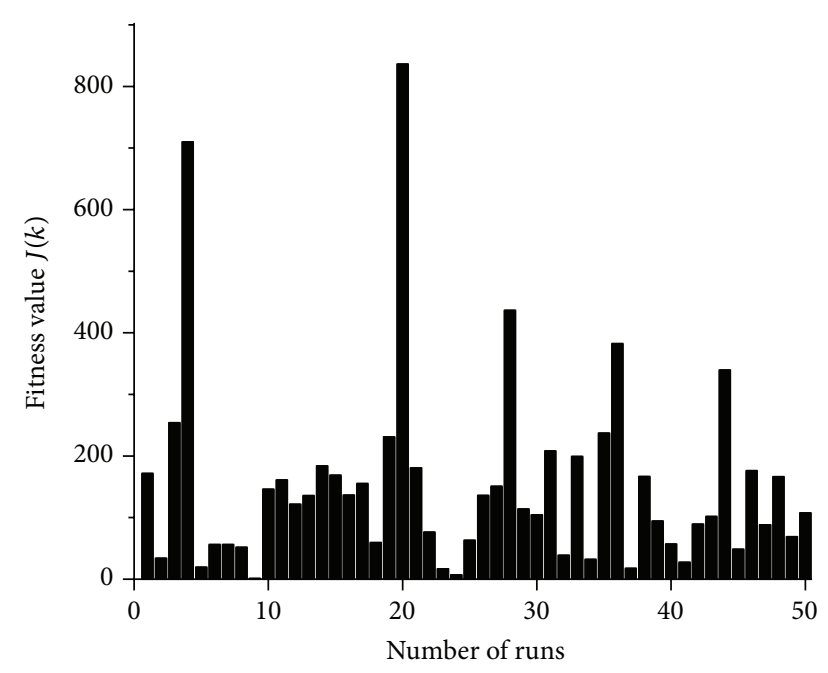

FIGURE 6: Fitness value versus number of runs (500 PHEVs).

Finally, Table 3 summarizes the result. From that it can be concluded that average best fitness remains almost in similar pattern for five (05) different scenarios.

\subsection{Performance Evaluation of GSA}

4.1.1. Convergence Analysis. It can be apparently seen that although the algorithm has been set to run for maximum 100 iterations, the convergence happened in about 20 iterations. The result derived in this paper reveals that each object of the standard GSA converges to a stable point. Here, the assumption was that the gravitational and inertia masses are the same. However, for some applications different values for them can be used. A heavier inertia mass provides a slower motion of agents in the search space and hence a more

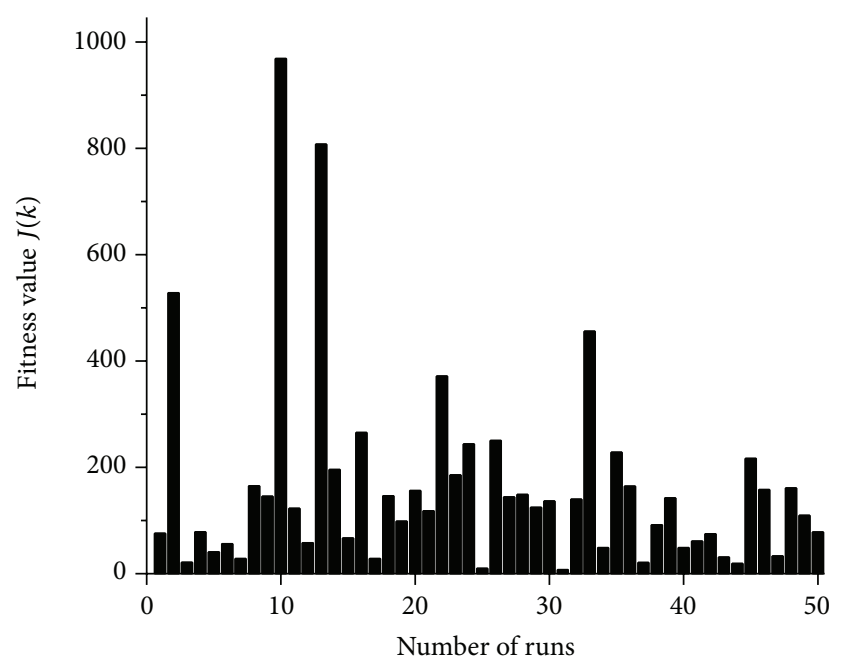

Figure 7: Fitness value versus number of runs (for 1000 PHEVs).

precise search [20]. On the contrary, a heavier gravitational mass causes a higher attraction of agents. This allows a faster convergence. The analysis results confirm the convergence characteristics of GSA according to the given parameters ranges of the algorithm. Figures 8, 9, 10, 11, and 12 show the convergence behavior of GSA. The best fitness function shows convergences after the same iterations (35 iterations) for both 50 and 100 numbers of PHEVs while for 500 and 1000 numbers of PHEVs, it shows early convergence (before 20 iterations).

4.1.2. Robustness. The similar numeric patterns of average best fitness show the robustness of GSA method. This method resists change without adapting its initial stable configuration 
TABLE 3: Fitness evaluation of GSA.

\begin{tabular}{|c|c|c|c|c|c|}
\hline $\begin{array}{l}\text { Fitness function } \\
J(k)\end{array}$ & For 50 PHEVs & For 100 PHEVs & For 300 PHEVs & For 500 PHEVs & For 1000 PHEVs \\
\hline Maximum best fitness & 781.1267 & 579.3955 & 743.1251 & 836.2707 & 968.7652 \\
\hline Average best fitness & 158.8289 & 139.7536 & 172.4296 & 152.36437 & 161.52349 \\
\hline Minimum best fitness & 0.2191 & 3.2523 & 2.3279 & 0.9818 & 7.2747 \\
\hline
\end{tabular}

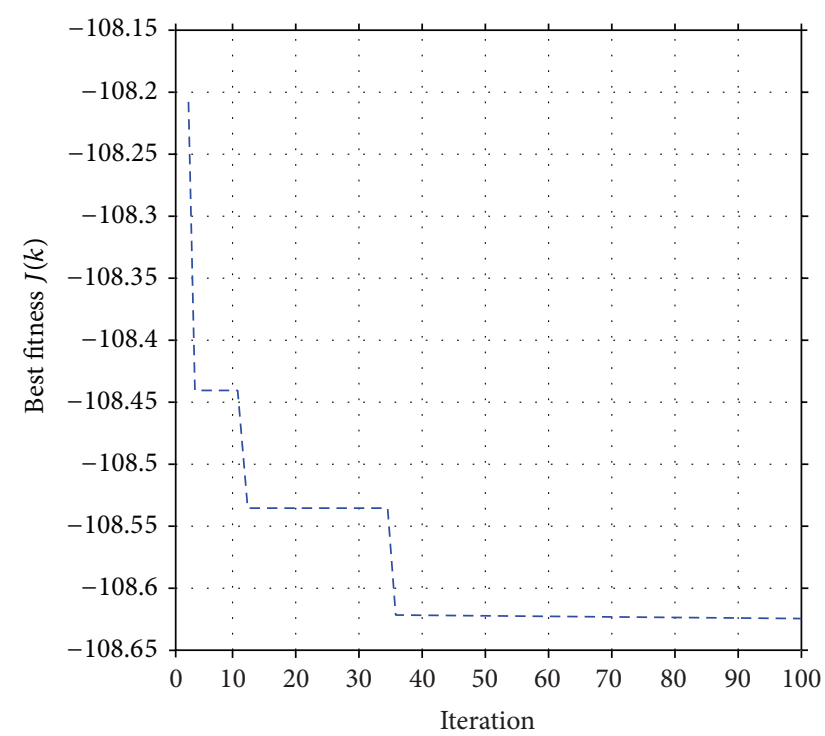

FIGURE 8: Best fitness versus iteration (50 PHEVs).

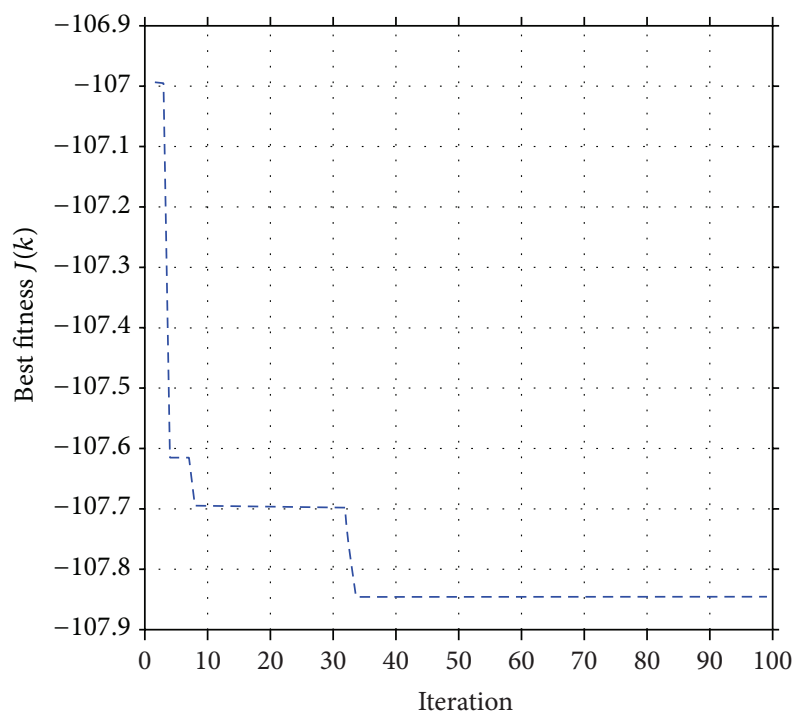

FIgURE 9: Best fitness versus iteration (100 PHEVs).

for different cases (number of PHEVs) which proves GSA as a robust algorithm.

4.1.3. Diversity. Here, the average best fitness gives different values with the increment of PHEVs population. The rate of convergence of mass agents in GSA is good through the

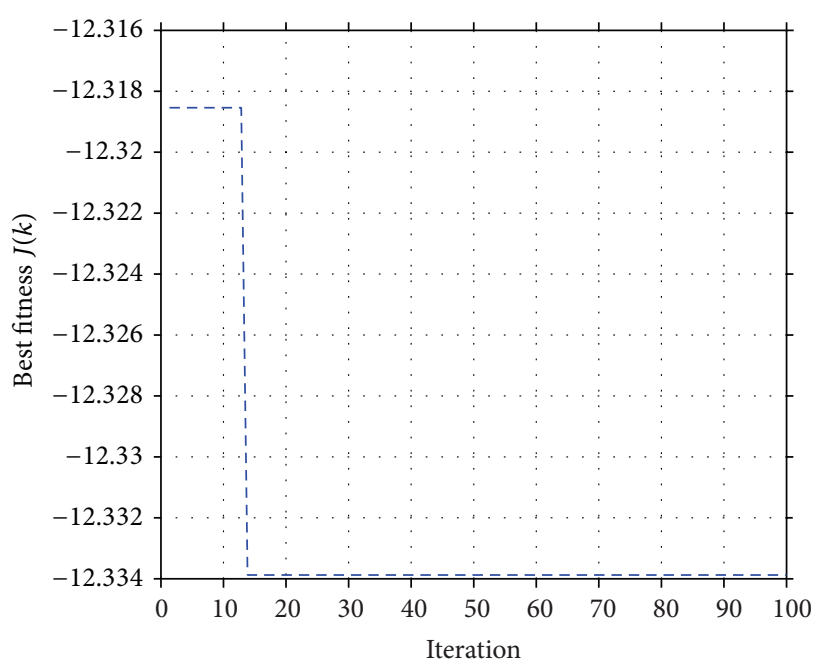

FIgUre 10: Best fitness versus iteration (300 PHEVs).

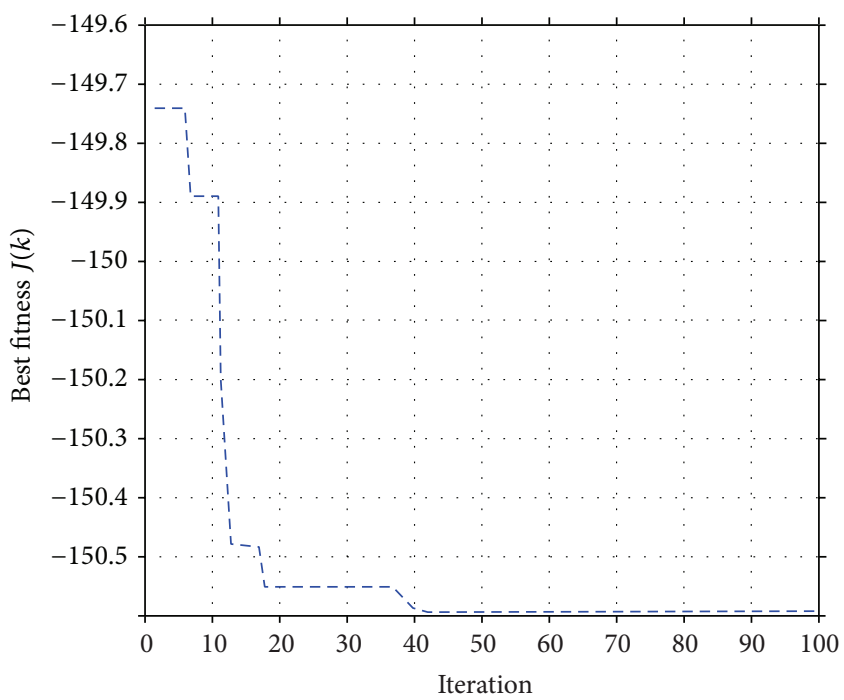

FIgURE 11: Best fitness versus iteration (500 PHEVs).

fast information flowing among mass agents, so its diversity decreases very quickly in the successive iterations and leads to a suboptimal solution.

4.1.4. Computational Cost. Here, we measured the computational cost of the algorithm in terms of total running time. Table 6 shows the computational time of GSA for five different scenarios. Here, average CPU time is measured in seconds. 


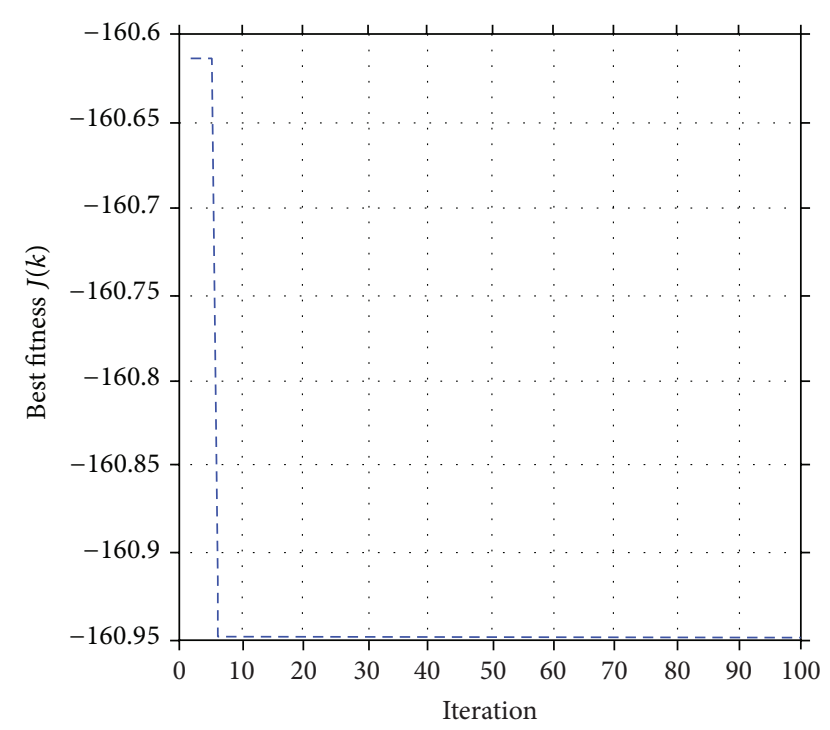

FIGURE 12: Best fitness versus iteration (1000 PHEVs).

As GSA needs a good number of parameter tuning, the computational cost increases with the increment of the total number of PHEVs.

4.1.5. Quality of Solution. When an algorithm finds an optimal solution to a given problem, one of the important factors is speed and rate of convergence to the optimal solution. For heuristics, the additional consideration of how close the heuristic solution comes to optimal is generally the primary concern of the researcher [32]. In GSA, the faster convergence and better exploitation rate ensure good quality solution, which is best fitness function.

For this optimization, the initial state of charge was expressed as a random number which is continuous and uniform between 0.2 and 0.6 . The sample time was set around 1200 seconds (20 minutes). The remaining charge time was defined as continuous random number between 0 and 6 hours. The price according to customer's choice for paying the bill for electricity was expressed as a continuous random number which is in between $\$ 1$ and $\$ 2$.

The capacity of the battery was assumed to be identical for all vehicles.

4.2. Comparison between GSA and PSO. Particle swarm optimization (PSO) with the parameter settings stated in Table 4 was also performed for the same objective function and compared with the performance of gravitational search algorithm in terms of average best fitness. The swarm size and maximum iterations were set exactly the same as those of GSA algorithm for the comparison purpose. The values of parameters $c 1, c 2$, and $w$ were set as standard values, 1.4, 1.4, and 0.9 , respectively.

From Figure 13 it is clear that gravitational search algorithm outperformed particle swarm optimization in terms of average best fitness. Starting from 50 numbers of PHEVs up to 1000 PHEVs, GSA shows better fitness value than PSO.
TABLE 4: PSO parameter settings.

\begin{tabular}{lc}
\hline Parameters & Values \\
\hline Size of the swarm & 100 \\
Maximum number of steps & 100 \\
PSO parameter, $c 1$ & 1.4 \\
PSO parameter, $c 2$ & 1.4 \\
PSO inertia $(w)$ & 0.9 \\
Maximum iteration & 100 \\
Number of runs & 50 \\
\hline
\end{tabular}

TABLE 5: Summarizes the comparisons of GSA with PSO algorithm in terms of average best fitness.

\begin{tabular}{lcc}
\hline Average best fitness for & PSO & GSA \\
\hline 50 PHEVs & 142.839 & 158.8289 \\
100 PHEVs & 171.102 & 182.3097 \\
300 PHEVs & 169.312 & 172.4296 \\
500 PHEVs & 150.869 & 152.36437 \\
1000 PHEVs & 156.802 & 161.52349 \\
\hline
\end{tabular}

TABLE 6: Computational time for PSO and GSA.

\begin{tabular}{lcc}
\hline Computational Time (sec.) & PSO & GSA \\
\hline 50 PHEVs & 1.650 & 2.721 \\
100 PHEVs & 1.686 & 4.439 \\
500 PHEVs & 1.990 & 18.165 \\
1000 PHEVs & 2.398 & 36.275 \\
\hline
\end{tabular}

Table 7 illustrates the advantages and disadvantages of both GSA and PSO for solving different optimization problems.

It has been proven that gravitational search algorithm has good ability to search for the global optimum, but it suffers from slow searching speed in the last iterations [35]. Moreover, the inertia mass is against the motion and slows the mass movement. Agents with heavy inertia mass move slowly and hence search the space more locally. So, it can be considered as an adaptive learning rate [34]. GSA is a memory-less algorithm. However, it works competently like the algorithms with memory. Our simulation results show the good convergence rate of the GSA.

\section{Conclusion and Recommendations}

In this paper, gravitational search algorithm- (GSA-) based optimization was performed in order to optimally allocate power to each of the PHEVs entering into the charging station. A sophisticated controller will need to be designed in order to allocate power to PHEVs appropriately. For this wake, the applied algorithm in this paper is a step towards real-life implementation of such controller for PHEV charging infrastructures.

Here, five (05) different numbers of PHEVs were considered for MATLAB ${ }^{\odot}$ simulation and then obtained results were compared with PSO in terms of average best fitness. The 
TABLE 7: Advantages and disadvantages of PSO and GSA.

\begin{tabular}{lcc}
\hline $\begin{array}{l}\text { Optimization } \\
\text { method }\end{array}$ & Advantages & Disadvantages \\
& Less parameters tuning \\
PSO & Easy constraint \\
& $\begin{array}{c}\text { Good for multiobjective } \\
\text { optimization } \\
{[33]}\end{array}$ & $\begin{array}{c}\text { Low quality solution } \\
\text { Needs memory to update velocity } \\
\text { Slow convergence rate }\end{array}$ \\
\hline GSA & High quality solution & Nood convergence rate \\
& Local exploitation capability $[34]$ & More parameters tuning \\
\hline
\end{tabular}

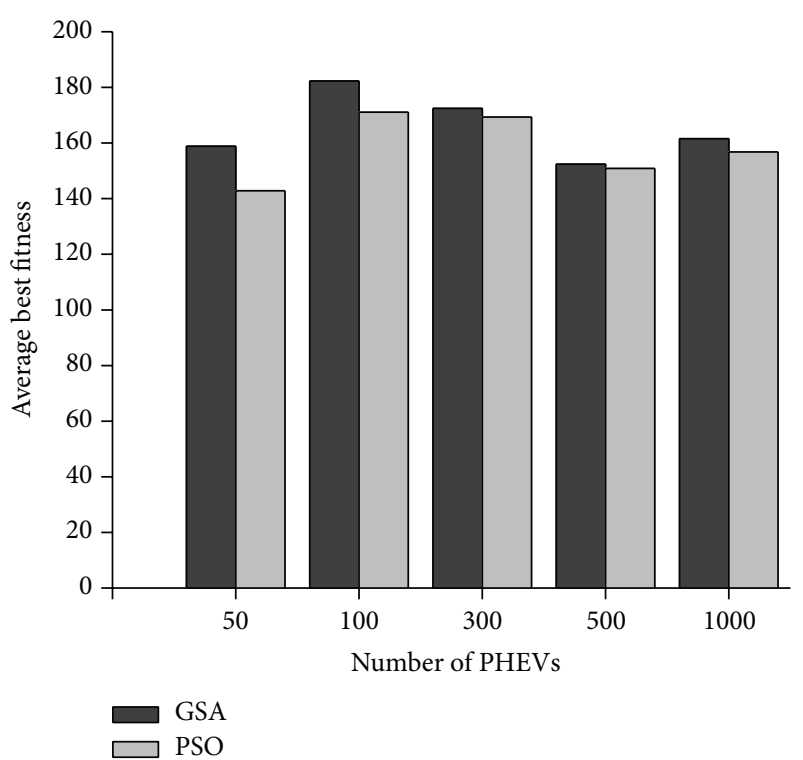

FIGURE 13: Average best fitness versus number of PHEVs.

success of the electrification of transportation sector solely depends on charging infrastructure. Only proper charging control and infrastructure management can assure the larger penetration of PHEVs. The researchers should try to develop efficient control mechanism for charging infrastructure in order to facilitate upcoming PHEVs penetration in highways. In future, more vehicles should be considered for intelligent power allocation strategy and improved versions of GSA and hybrid swarm intelligence based methods should be applied to ensure low computational cost.

\section{Nomenclature and Acronyms}

PHEVs: Plug-in hybrid electric vehicles

EPRI: Electric Power Research Institute

V2G: Vehicle to grid

SoC: State of charge

ICEVs: Internal combustion engine vehicles

AEVs: All-electric vehicles

HEVs: Hybrid electric vehicles

AER: All-electric range

$I_{i}(k)$ : Charging current over $\Delta t$
$C_{r, i}(k)$ : Remaining battery capacity required to be filled for $i$ th PHEV at time step $k$

$C_{i}$ : $\quad$ Rated battery capacity of the $i$ th PHEV (Farad)

$T_{r, i}(k)$ : Remaining time for charging the $i$ th PHEV at time step $k$

$D_{i}(k): \quad$ Price difference

$w_{i}(k)$ : Charging weighting term of the ith PHEV at time step

SoC $_{i}(k+1)$ : State of charge of the $i$ th PHEV at time step $k+1$

$\mathrm{SoC}_{i, \max }: \quad$ User-defined maximum battery SoC limit for the $i$ th PHEV

$P_{\text {utility }}: \quad$ Power available from the utility

$P_{i, \text { max }}: \quad$ Maximum power that can be absorbed by a specific PHEV

$\eta$

Overall charging efficiency of the charging station.

\section{Conflict of Interests}

The authors declare that there is no conflict of interests regarding the publication of this paper.

\section{Acknowledgments}

The authors would like to thank Universiti Teknologi Petronas for supporting the research under Graduate Assistance Scheme. This research paper is financially sponsored by the Centre of Graduate study with the support of the Department of Fundamental \& Applied Sciences, Universiti Teknologi Petronas.

\section{References}

[1] On certain integrals International Energy Agency (lEA), Transport, Energy and C02-Moving Towards Sustainability, 2009.

[2] D. Holtz-Eakin and T. M. Selden, "Stoking the fires? $\mathrm{CO}_{2}$ emissions and economic growth," Journal of Public Economics, vol. 57, no. 1, pp. 85-101, 1995.

[3] S. F. Tie and C. W. Tan, "A review of energy sources and energy management system in electric vehicles," Renewable and Sustainable Energy Reviews, vol. 20, pp. 82-102, 2013.

[4] "Environmental assessment of plug-in hybrid electric vehicles, Volume 1: Nationwide greenhouse gas emissions," Tech. Rep. 
1015325, Electric Power Research Institute (EPRI), Palo Alto, Calif, USA, 2007.

[5] H. Lund and W. Kempton, "Integration of renewable energy into the transport and electricity sectors through V2G," Energy Policy, vol. 36, no. 9, pp. 3578-3587, 2008.

[6] A. R. Hota, M. Juvvanapudi, and P. Bajpai, "Issues and solution approaches in PHEV integration to the smart grid," Renewable and Sustainable Energy Reviews, vol. 30, pp. 217-229, 2014.

[7] J. Soares, T. Sousa, H. Morais, Z. Vale, B. Canizes, and A. Silva, "Application-Specific Modified Particle Swarm Optimization for energy resource scheduling considering vehicle-to-grid," Applied Soft Computing Journal, 2013.

[8] W. Su and M.-Y. Chow, "Computational intelligence-based energy management for a large-scale PHEV/PEV enabled municipal parking deck," Applied Energy, vol. 96, pp. 171-182, 2012.

[9] W. Su and M. Y. Chow, "Performance evaluation of a PHEV parking station using particle swarm optimization," in Proceedings of the IEEE PES General Meeting, pp. 1-6, July 2011.

[10] W. Su and M.-Y. Chow, "Performance evaluation of an EDAbased large-scale plug-in hybrid electric vehicle charging algorithm," IEEE Transactions on Smart Grid, vol. 3, no. 1, pp. 308315, 2012.

[11] K. Morrow, D. Karner, and J. Francfort, "Plug-in hybrid electric vehicle charging infrastructure review," Tech. Rep., The Idaho National Laboratory, 2008.

[12] "Investigation into the scope for the transport sector to switch to electric vehicles and plug- in hybrid vehicles," Tech. Rep., Department for Transport, London, UK, 2008.

[13] D. Mayfield, "Site Design for Electric Vehicle Charging Stations, ver. 1.0," Sustainable Transportation Strategies, July 2012.

[14] G. Boyle, Renewable Electricity and the Grid: The Challenge of Variability, Earthscan Publications, London, UK, 2007.

[15] A. Hess, F. Malandrino, M. B. Reinhardt, C. Casetti, K. A. Hummel, and J. M. Barceló-Ordinas, "Optimal deployment of charging stations for electric vehicular networks," in Proceedings of the 1st ACM Conference on Urban Networking (UrbaNe '12), pp. 1-6, New York, NY, USA, December 2012.

[16] Z. Li, Z. Sahinoglu, Z. Tao, and K. H. Teo, "Electric vehicles network with nomadic portable charging stations," in Proceeding of the IEEE 72nd Vehicular Technology Conference Fall (VTC '10), pp. 1-5, Ottawa, Canada, September 2010.

[17] T. Ghanbarzadeh, S. Goleijani, and M. P. Moghaddam, "Reliability constrained unit commitment with electric vehicle to grid using hybrid particle swarm optimization and ant colony optimization," in Proceeding of the IEEE PES General Meeting: The Electrification of Transportation and the Grid of the Future, pp. 1-7, San Diego, Calif, USA, July 2011.

[18] A. Shafiei and S. S. Williamson, "Plug-in hybrid electric vehicle charging: current issues and future challenges," in Proceedings of the IEEE Vehicle Power and Propulsion Conference (VPPC '10), pp. 1-8, September 2010.

[19] J. Yan, C. Li, G. Xu, and Y. Xu, "A novel on-line self-learning state-of-charge estimation of battery management system for hybrid electric vehicle," in Proceedings of the IEEE Intelligent Vehicles Symposium, pp. 1161-1166, June 2009.

[20] E. Rashedi, H. Nezamabadi-pour, and S. Saryazdi, "GSA: a gravitational search algorithm," Information Sciences, vol. 179, no. 13, pp. 2232-2248, 2009.

[21] D. Martens, B. Baesens, and T. Fawcett, "Editorial survey: swarm intelligence for data mining," Machine Learning, vol. 82, no. 1, pp. 1-42, 2011.
[22] J. Krause, J. Cordeiro, R. S. Parpinelli, and H. S. Lopes, "A survey of swarm algorithms applied to discrete optimization problems," in Swarm Intelligence and Bio-inspired Computation: Theory and Applications, pp. 169-191, Elsevier Science \& Technology Books, 2013.

[23] O. Okobiah, S. P. Mohanty, and E. Kougianos, "Geostatistics inspired fast layout optimization of nanoscale CMOS phase locked loop," in Proceedings of the 14th International Symposium on Quality Electronic Design (ISQED '13), pp. 546-551, Santa Clara, Calif, USA, March 2013.

[24] W.-Y. Chang, "The state of charge estimating methods for battery: a review," ISRN Applied Mathematics, vol. 2013, Article ID 953792, 7 pages, 2013.

[25] T. Ganesan, I. Elamvazuthi, K. Z. Ku Shaari, and P. Vasant, "Swarm intelligence and gravitational search algorithm for multi-objective optimization of synthesis gas production," Applied Energy, vol. 103, pp. 368-374, 2013.

[26] O. T. Altinoz and A. E. Yilmaz, "Calculation of optimized parameters of rectangular patch antenna using gravitational search algorithm," in Proceedings of the International Symposium on INnovations in Intelligent SysTems and Applications (INISTA '11), pp. 349-353, IEEE, June 2011.

[27] Ö. T. Altinöz, A. E. Yilmaz, and G. W. Weber, "Orthogonal array based performance improvement in the gravitational search algorithm," Turkish Journal of Electrical Engineering and Computer Sciences, vol. 21, no. 1, pp. 174-185, 2013.

[28] N. M. Sabri, M. Puteh, and M. R. Mahmood, "A review of gravitational search algorithm," International Journal of Advances in Soft Computing and Its Applications, vol. 5, no. 3, pp. 1-39, 2013.

[29] R. E. Haskell, G. Castelino, and B. Mirshab, "Efficient algorithm for locating the global maximum of an arbitrary univariate function," Journal of Forth Application and Research, vol. 5, no. 3, pp. 357-364, 1989.

[30] M. Udgir, H. M. Dubey, and M. Pandit, "Gravitational search algorithm: a novel optimization approach for economic load dispatch," in Proceedings of the Annual International Conference on Emerging Research Areas and International Conference on Microelectronics, Communications and Renewable Energy (AICERA/ICMiCR '13), pp. 1-6, 2013.

[31] A. A. Bazmi and G. Zahedi, "Sustainable energy systems: role of optimization modeling techniques in power generation and supply-a review," Renewable and Sustainable Energy Reviews, vol. 15, pp. 3480-3500, 2011.

[32] R. S. Barr, B. L. Golden, J. P. Kelly, M. G. C. Resende, and W. R. Stewart Jr., "Designing and reporting on computational experiments with heuristic methods," Journal of Heuristics, vol. 1, no. 1, pp. 9-32, 1995.

[33] Y.-T. Kao and E. Zahara, "A hybrid genetic algorithm and particle swarm optimization for multimodal functions," Applied Soft Computing, vol. 8, pp. 849-857, 2008.

[34] I. Rahman, P. M. Vasant, B. S. M. Singh, and M. AbdullahAl-Wadud, "Intelligent energy allocation strategy for PHEV charging station using gravitational search algorithm," AIP Conference Proceedings, vol. 1621, pp. 52-59, 2014.

[35] S. Mirjalili, S. Z. Mohd Hashim, and H. Moradian Sardroudi, "Training feedforward neural networks using hybrid particle swarm optimization and gravitational search algorithm," Applied Mathematics and Computation, vol. 218, no. 22, pp. 11125-11137, 2012. 


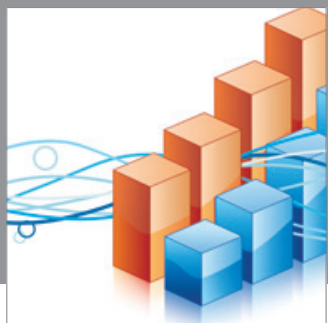

Advances in

Operations Research

mansans

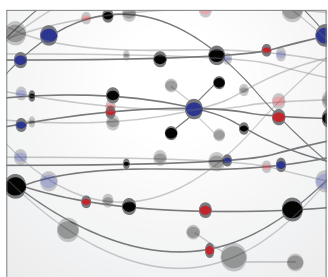

The Scientific World Journal
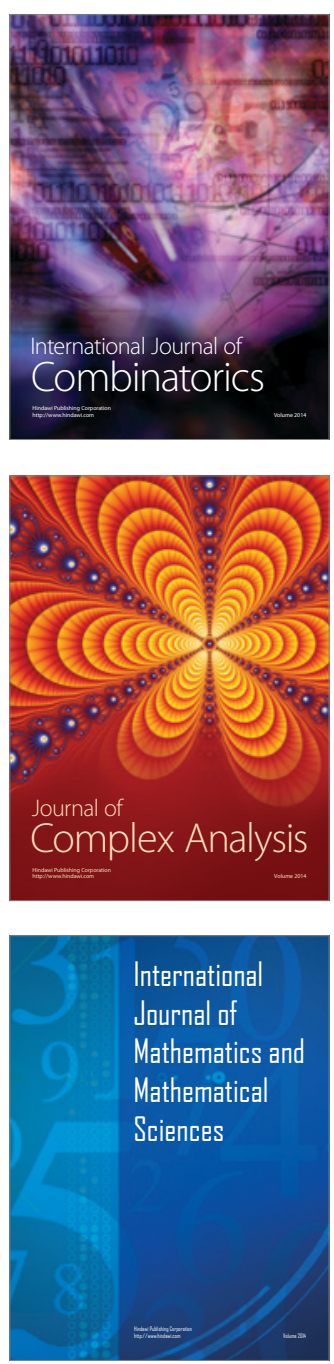
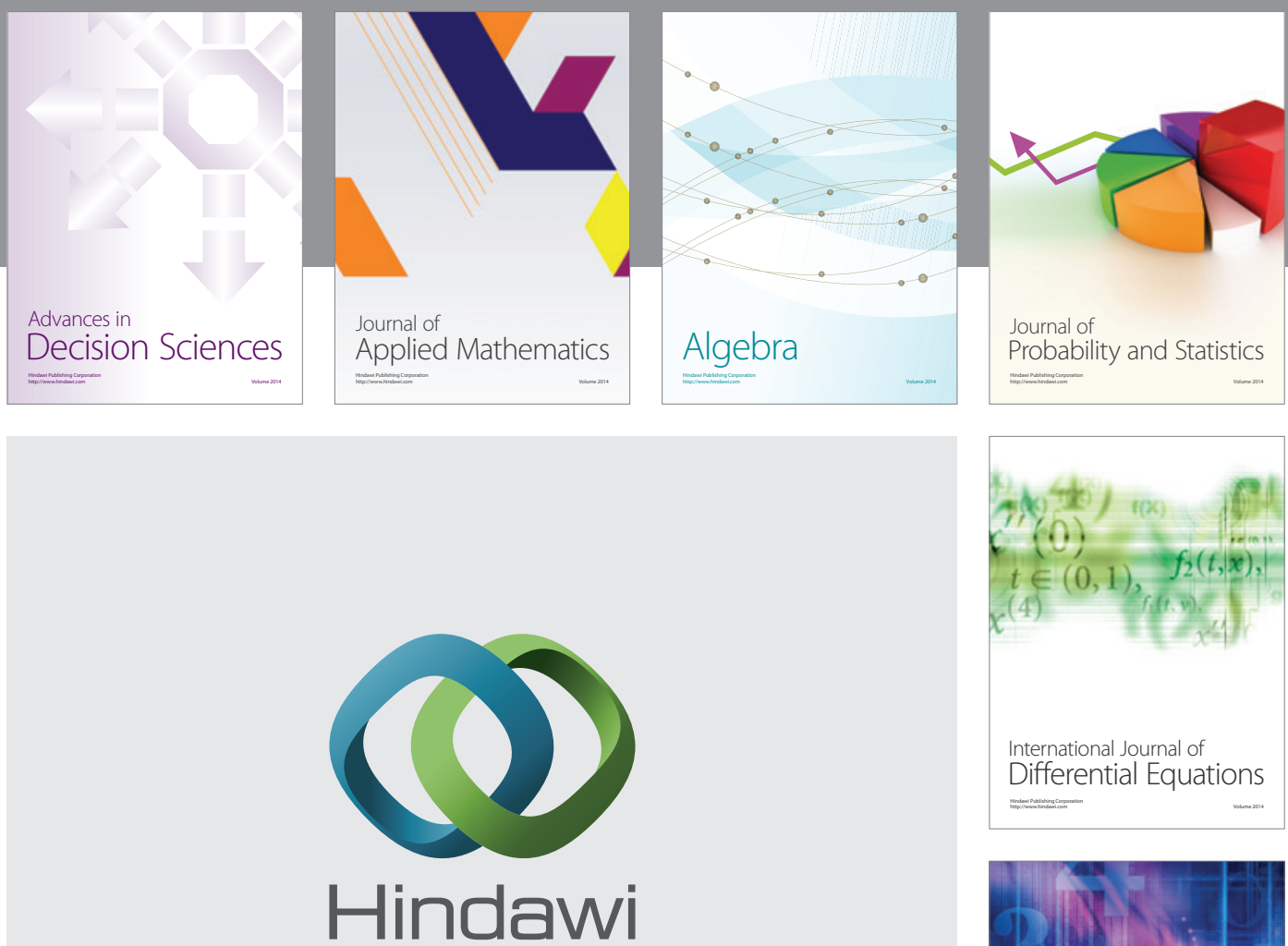

Submit your manuscripts at http://www.hindawi.com
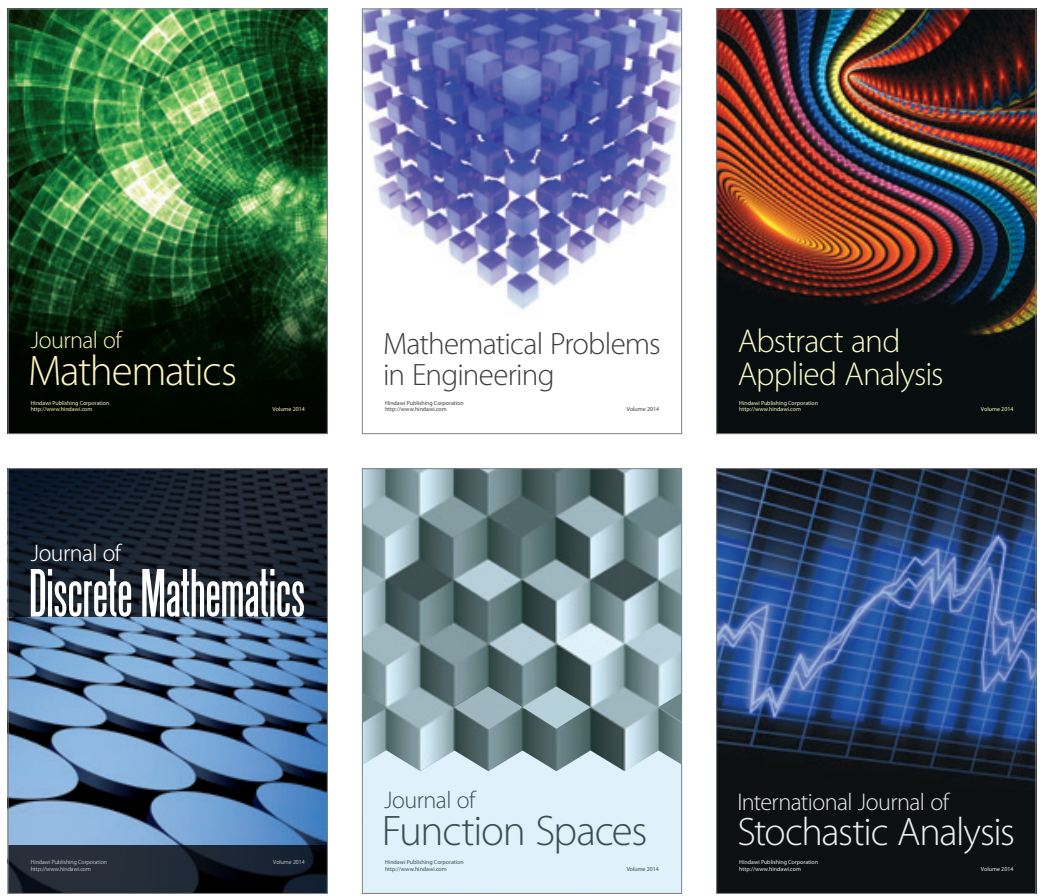

Journal of

Function Spaces

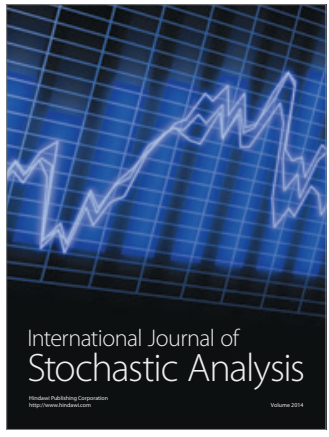

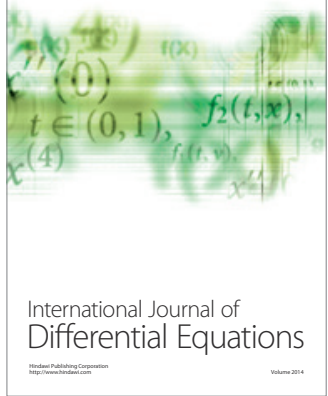
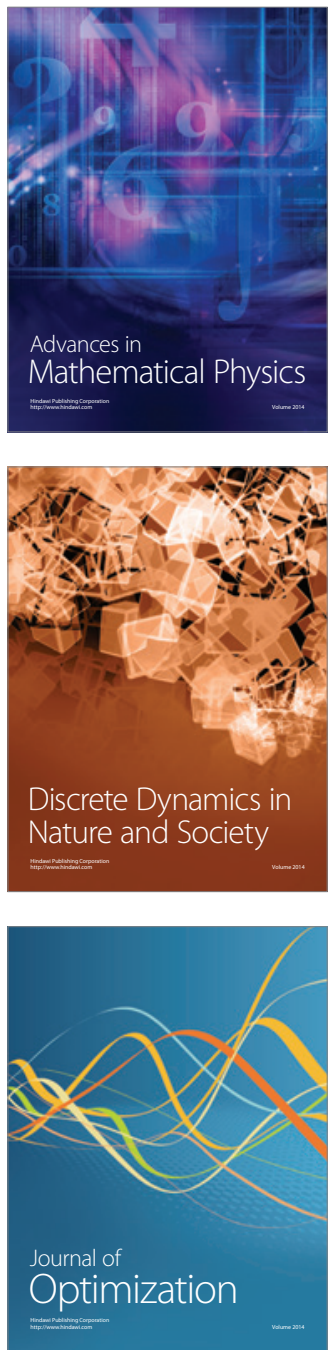